\title{
Editorial: Special Issue on "QoE Monitoring and Management for Future Internet Media Services"
}

\author{
Tasos Dagiuklas ${ }^{1} \cdot$ Raimund Schatz ${ }^{2}$. \\ Pedro Assuncao $^{3}$ - Luigi Atzori ${ }^{4}$
}

Published online: 21 September 2017

(C) Springer Science+Business Media, LLC 2017

The effectiveness of distributed multimedia applications and mobile computing services as they have become dominant in the modern telecommunication era, is strongly based on the performance of the communication systems that deliver content to the end-user. However, it is a fact that the aforementioned effectiveness of the presentation of any multimedia service is ultimately measured by the end-user's experience and the resulting levels of delight and annoyance.

In this context, the concept Quality of Experience (QoE) has become a central reference point as it describes the overall acceptability of an application or service strictly from the user's point of view. It is a subjective measure of end-to-end service performance from the user perspective as well as an indication of how well the system meets the user's needs. Encompassing many different aspects, QoE rivets on the true feelings of end users when they watch streaming video and podcasts, listen to digitized music and browse the Internet relying on a plethora of methods and devices. For these reasons, researchers and practitioners alike have started to integrate QoE in the design of quality evaluation models for end-user devices, resource management of network infrastructures and services in order to enable new and better multimedia experiences - a trend that will be accelerated by the evolution of the Future Internet and $5 \mathrm{G}$ Communications.

The special issue consists of four papers that cover different aspects of QoE in terms of QoE Management, QoE Monitoring in Future Internet Scenarios, and machine-learning based QoE Modeling.

The first paper is entitled "A Remote Display QoE Improvement Scheme for Interactive Applications in Low Network Bandwidth Environment” (https://doi.org/10.1007/s11042017-4692-z), by Ngo Quang Thai, Md. Abu Layek, Pham Xuan Qui and Seungkyu Lee.

Tasos Dagiuklas

tdagiuklas@1sbu.ac.uk

1 London South Bank University, London, UK

2 AIT Austrian Institute of Technology, Vienna, Austria

3 Institute of Telecommunications/IPL, Leiria, Portugal

4 University of Cagliari, Cagliari, Italy 
The authors propose a novel QoE scheme that dynamically controls the quality setting of the image compression before the screen transmission to decrease response time of the system while maintaining the satisfactory image quality. The proposed scheme selects the best quality for the current network bandwidth, then it uses the remaining bandwidth to improve the quality setting of low motion regions without any adverse effect on response time. Both quantitative measurements and users' evaluations have demonstrated QoS and QoE improvements.

The second paper is entitled "Challenges of Future Multimedia QoE Monitoring for Internet Service Providers" (https://doi.org/10.1007/s11042-017-4870-z) by Werner Robitza, Arslan Ahmad, Péter András Kara, Luigi Atzori, Maria Martini, Alexander Raake and Lingfen Sun. The authors first provide an overview of state-of-the-art quality monitoring models, probing technologies and architectures. The main part is devoted to a discussion of the challenges of monitoring QoE for Internet Service Providers, including user privacy, application-layer encryption and use of virtualized networks.

The third paper is entitled "A Machine Learning Approach to Classifying YouTube QoE Based on Encrypted Network Traffic" (https://doi.org/10.1007/s11042-017-4728-4) by Irena Orsolic, Dario Pevec, Mirko Suznjevic and Lea Skorin-Kapov. The authors present a methodology for the classification of end users' QoE when watching YouTube videos, based only on statistical properties of encrypted network traces. On behalf of collected network data, machine learning models have been developed for video quality classification. The authors show that classification accuracy is up to $84 \%$ when using three quality classes (low, medium and high) and up to $91 \%$ when using binary classification (low and high).

The fourth paper entitled "Unsupervised Deep Learning for Real-Time Assessment of Video Streaming Services” (https://doi.org/10.1007/s11042-017-4831-6) by Maria Torres Vega, Decebal Constantin Mocanu and Antonio Liotta proposes a light-weight No Reference (NR) quality estimation method that employs unsupervised machine learning techniques highly suitable for QoE monitoring application. The proposed method makes use of the excellent density estimation capabilities of restricted Boltzmann machines that process lightweight video features computed on quality-degraded videos. The approach has been successfully tested on two network impaired video data sets, the LIMP and the ReTRiEVED video quality databases.

We wish to thank all authors for considering this special issue of the Multimedia Tools and Applications Journal as a venue for submitting their papers. We express our gratitude to all reviewers who devoted their precious time in providing valuable feedback on all the papers considered for this special issue. Of the 10 papers submitted, 4 were accepted after two rounds of review.

Finally, we thank the Editor-in-Chief, Professor Borko Furht, for his great support and encouragements. We would also like to take this opportunity to thank the staff of the Editorial Office at Springer for his unconditional support and time throughout the preparation of this issue.

We hope that this special issue will provide a valuable source of reference for researchers, designers, and engineers working in the area of multimedia networking and content provisioning. 